\title{
SU ALCUNE PROPRIETÀ NOTEVOLI DEL ROTORE DEL VENTO DI GRADIENTE
}

\author{
Felice Di Benedetto
}

1. Introduzione. - Nonostante clue il concetto di rotore impermei tutta la cinematica dell'almosfera, il campo di questa grandezza vettoriale si presenta tuttora molto complicato e sarebbe, pertanto, molto importante e di notevole interesse tentare di gettare un po' di luce su questo problema, analizzando i diversi fattori elementari che compongono il rotore e che possono, però, essere determinati singrolarmente dalle carte meteorologiche. Lo scopo del presente lavoro è quello di mettere in rilievo questi diversi fattori, per valutarne la influenza singola e reciproca e chiarire alcuni dei punti più oscuri di questo importante ramo della cinematica dell'atmosfera.

2. Proprietà fondamentali e definizioni. - Prima di addentrarci in questo studio, sarà opportuno ricordare le proprietà fondamentali del simbolo hamiltoniano $\nabla$, denominato "ascendente" o "nabla ".

Questo simbolo è un operatore (differenziale) che ha per " proiezioni " sugli assi di riferimento i tre simboli delle derivate parziali $\partial / \partial x, \partial / \partial y, \partial / \partial z$, rispettivamente.

Se con $f$ indichiamo una grandezza scalare (pressione, temperatura, ecc.), funzione delle tre cocrdinate $x, y, z$, il veltore $\nabla f$ ammelle per proiezioni $\partial f / \partial x, \partial f / \partial y$, e $\partial f / \partial z$. Per conseguenza, il vettore gradiente della funzione scalare $f$, di cui le proiezioni sono, per definizione, $-\partial f / \partial x,-\partial f / \partial y,-\partial f / \partial z$, ̀̀ rappresentato da $-\nabla f$. Sicché:

$$
\operatorname{grad} f=-\nabla f
$$

Sia $\bar{v}$ un vettore (qualunque), di componenti $u, v, w$. Formiamo il prodotto vettoriale $\nabla \wedge \bar{v}$. Le proiezioni (componenti) sugli assi $x, y$ e $z$ di questo veltore sono, come è noto, i determinanti (coi loro 
segni) contenuti nella matrice formata con le proiezioni dei vettori dati. Quindi :

$$
\nabla \wedge \bar{v}=\left\{\begin{array}{l}
\frac{\partial w}{\partial y}-\frac{\partial v}{\partial z} \\
\frac{\partial u}{\partial z}-\frac{\partial w}{\partial x} \\
\frac{\partial v}{\partial x}-\frac{\partial u}{\partial y}
\end{array}\right.
$$

Queste componenti sono anche le proiezioni del vettore rot $\bar{v}$, quindi possiamo scrivere:

$$
\operatorname{rot} \bar{v}=\nabla \wedge \bar{v}
$$

Converremo di chiamare il rotore "ciclonico", quanclo la sua grandezza $\mid$ rot $\boldsymbol{v} \mid>0$ e "anticiclonico", quando $\mid$ rot $\boldsymbol{v} \mid<0$. Queste denominazioni derivano dal fatto che, quando la grandezza del rotore è positiva, il moto del fluido è rotazionale ciclonico e, quando tale grandezza è negativa, il moto è rotazionale anticiclonico.

Ricordiamo ancora delle relazioni che saranno utili in seguito, tenendo presente che il nahla opera su grandezze scalari.

Siano $f$ e $g$ due funzioni (scalari) di $x, y$ e $z$. Si ha:

$$
\nabla(f+g)=\nabla f+\nabla g
$$

Analogamente :

$$
\nabla(f g)=f \nabla g+g \nabla f
$$

In particolare, quando $f=g$,

$$
\nabla f^{2}=2 f \cdot \nabla f
$$

e

$$
\nabla \frac{f}{g}=\frac{\nabla_{j}^{f}}{g}-\frac{t}{g^{2}} \nabla g
$$

Consideriamo ora il prodotto vettoriale di $\square$ per il vettore $f \cdot \bar{v}$, dove $f$ è una funzione scalare di $x, y$, $z$. 
Si ha:

$$
\nabla \wedge f \bar{v}==\left\{\begin{array}{l}
\frac{\partial}{\partial y}(\underline{t} w)-\frac{\partial}{\partial z}(f v) \\
\frac{\partial}{\partial z}(f u)-\frac{\partial}{\partial x}(f v) \\
\frac{\partial}{\partial x}(f v)-\frac{\partial}{\partial y}(f u)
\end{array}\right.
$$

Effettuando le derivazioni e riordinando, si vede facilmente che vale la seguente relazione:

$$
\nabla \wedge f \bar{v}=f \nabla \wedge \bar{v}-\bar{v} \wedge \nabla f
$$

In modo del tutto analogo, si può dimostrare che:

$$
\nabla \wedge(\bar{u} \wedge \bar{v})=(\nabla \times \bar{v}) \bar{u}-(\nabla \times \bar{u}) \bar{v}+\bar{v} \times \nabla \bar{u}-\bar{u} \times \nabla \bar{v}
$$

c, infine :

$$
\nabla \times f \bar{v}=f \nabla \times \bar{v}+\nabla f \times \bar{v}
$$

3. Calcolo del rotore del vento di gradiente. - Sia $v$ la grandezza della velocita del vento di gradiente, $R$ il raggio di curvatura della traiettoria, che, in prima approssimazione, faremo coincidere con l'isobara, $\varrho$ la densità dell'aria, $l$ il parametro di Coriolis $2 w$ sen $\varphi$ (dove $w$ è la velocità angolare della terra e $p$ la latitudine), $\bar{G}$ il vettore gradiente barico orizzontale di grandezza $G$ e $\bar{n}$ il versore normale alla traiettoria. L'equazione del vento di gradiente si scrive, allora, come segue:

$$
v\left(l \pm \frac{v}{R}\right) \bar{n}=\frac{G}{\varrho} \bar{n}=\frac{\bar{G}}{\varrho}
$$

Il segno positivo, come si sa, corrisponde ad una traiettoria ciclonica ed il segno negativo ad una traiettoria anticiclonica.

Sia $\bar{t}$ il versore della tangente alla traiettoria e $\bar{k}$ il versore verticale, che forma con $\bar{t}$ e $\bar{n}$ una terna levogira. Per far comparire il vettore velocità $\bar{v}$ nella [11] moltiplichiamo i due membri di questa espressione vettorialmente per il versore $\bar{k}$.

Si ottiene:

$$
v\left(l \pm \frac{\underline{y}}{\bar{K}}\right) \bar{n} \wedge \bar{k}=\frac{\bar{G}}{\bar{\varphi}} \wedge \bar{k}
$$


e, poiché

$$
\bar{n} \wedge \bar{k}=\bar{t}
$$

e

$$
v \bar{t}=\bar{v}
$$

si ha l'equazione richiesta

$$
\left(l \pm \frac{v}{R}\right) \bar{v}=\frac{\bar{G}}{\varrho} \wedge \bar{k}
$$

Moltiplicando vettorialmente per $\rightarrow 7$ i due membri della [12] si fa comparire il rotore del vettore $\bar{v}$. Infatti, dalla relazione:

$$
\nabla \wedge\left[\left(l \pm \frac{v}{R}\right) \bar{v}\right]=\nabla \wedge\left(\frac{\bar{G}}{\varrho} \wedge \bar{k}\right)
$$

sviluppando il primo membro con la [8], dove $f=l-\frac{v}{K}$, si ottiene:

$$
\nabla \wedge\left[\left(l \pm \frac{v}{R}\right) \bar{v}\right]=\left(l \pm \frac{v}{R}\right) \nabla \wedge \bar{v}-\bar{v} \wedge \nabla\left(l \pm-\frac{v}{R}\right)=\nabla \wedge\left(\frac{\bar{G}}{\varrho} \wedge \bar{k}\right)
$$

Da quest'ultima relazione si ha, quindi :

$$
\left(l \pm \frac{v}{R}\right) \nabla \wedge \bar{v}=\bar{v} \wedge \nabla\left(i \pm \frac{v}{R}\right)+\nabla \wedge\left(\frac{G}{\varrho} \wedge \bar{k}\right)
$$

La [4], applicata al primo termine del secondo membro, dạ infine :

$$
\left(l \pm \frac{v}{R}\right) \nabla \wedge \bar{v}=\bar{v} \wedge \nabla l \pm \bar{v} \wedge \nabla \frac{v}{k}+\nabla \wedge\left(\frac{\bar{i}}{\varrho} \wedge \bar{k}\right)
$$

Ora, poiché la velocità $\bar{v}$ del vento di gradiente dipende da quattro variabili $(l, R, G$ e $\varrho)$, è utile trasformare la [13], per far comparire in essa $i$ vettori gradienti di queste quattro variabili, ossia, $-\nabla l,-\nabla R,-\nabla G$ e $-\nabla \rho$.

Trasformiamo in primo luogo $\nabla v / R$.

Scriviamo, perciò, l'equazione del vento di gradiente nella forma:

$$
\pm\left(\frac{v}{R}\right)^{2}+\geq \frac{v}{R}=\frac{G}{\varrho R}
$$


Applicando il nabla alla [14] e utilizzando le formule: [4], [5], [6] e [7] si ottiene:

$$
\vdots 2 \frac{v}{R} \cdot \nabla \frac{v}{R}+l \nabla \frac{v}{R}+\frac{v}{R} ? l=\frac{1}{R} \nabla \frac{C^{\prime}}{\varrho}-\frac{G}{\varrho R^{2}} \nabla R
$$

Da questa risulta, dunque:

$$
\nabla \frac{v}{R}=\frac{\frac{1}{R} \nabla \frac{G}{0}-\frac{v}{R} \nabla l-\frac{G}{\varrho R^{2}} \nabla R}{l_{ \pm} 2 \frac{v}{R}}
$$

Ma, poiché

$$
\frac{G}{\varrho}=v\left(l \pm-\frac{v}{R}\right)
$$

si ottiene, infine:

$$
\nabla \frac{v}{R}=\frac{\frac{l}{R} \nabla \frac{G}{\varrho}-\frac{v}{K}-\nabla l-\frac{v}{R^{*}}\left(l \pm \frac{v}{R}\right) \nabla R}{l-2 \frac{v}{R}}
$$

Ora, osserviamo che al secondo membro della [13] compare il termine

$$
\pm \bar{v} \wedge \nabla \frac{v}{R}
$$

Quindi, moltipicando la [16] vettorialmente per $i \bar{v}$, si ha:

$$
\begin{gathered}
\pm \bar{v} \wedge \nabla \frac{v}{R}=-\frac{1}{R\left(l \pm 2 \frac{v}{R}\right)} \bar{v} \wedge \nabla \frac{G}{\varrho} \mp \frac{v}{R}\left(\frac{1}{l \pm 2 \frac{v}{R}}\right) \bar{v} \wedge \nabla l \mp \\
\mp \frac{v}{R}\left(\frac{l \pm \frac{v}{R}}{l+2 \frac{v}{R}}\right) \bar{v} \wedge R
\end{gathered}
$$


Sostituendo la [17] nella [13] e riducendo ( $\left.{ }^{1}\right)$, si ottiene la formula cercata:

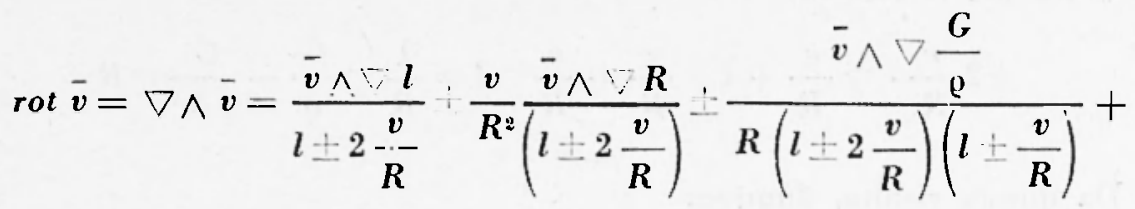

$$
\begin{aligned}
& +\frac{\forall \wedge\left(\frac{\bar{G}}{\varrho} \wedge \bar{k}\right)}{l \div \frac{v}{R}}
\end{aligned}
$$

Lo studio dei singoli termini al secondo membro della [18] ci permette di analizzare $i$ diversi fattori che compongono il rotore del vento di gradiente. $\dot{E}$ necessario tener presente che fino ad ora non è stata fatta alcuna ipotesi riguardante la forma del campo barico, per cui la [18] ha validità del tutto generale per i flussi di gradiente.

4. Effetto latitudine. - Il primo termine al secondo membro della [18]

$$
\frac{\bar{v} \wedge \vee l}{l \pm 2 \frac{v}{R}}
$$

rappresenta l'effetto della latitudine sul rotore del vento di gradiente. Questo termine vale per le depressioni e per grli anticicloni, con l'avvertenza di prendere il segno positivo del denominatore per le depressioni ed il segno negativo per gli anticicloni.

(1) Infatti, nella riduzione si ha:

$$
\begin{aligned}
\frac{\bar{v} \wedge \nabla l}{l \pm \frac{v}{K}} & =\frac{v}{K}\left[\frac{\bar{v} \wedge}{\left(l \pm \frac{v}{R}\right)} \frac{\nabla l}{\left(l \pm 2 \frac{v}{R}\right)}\right]=\frac{\bar{v} \wedge \nabla l}{l+\frac{v}{R}}\left[1 \pm \frac{v}{R\left(l \pm 2 \frac{v}{K}\right)}\right]= \\
& =\frac{\bar{v} \wedge \nabla l}{l \pm \frac{v}{K}}\left[\frac{R l+2 v+v}{R\left(l \pm 2 \frac{v}{K}\right)}\right]=\frac{\bar{v} \wedge \nabla l}{l \pm \frac{v}{K}}\left[\frac{R l-v}{R\left(l \pm 2 \frac{v}{R}\right)}\right]= \\
& =\frac{\bar{v} \wedge \nabla l}{l \pm 2 \frac{v}{K}}
\end{aligned}
$$


Questo effetto puì essere rappresentato analiticamente come segue: Per fissare le idee, sia $p$ un'isolora appartenente ad una depressione (fig. 1).

Di un punto $M$ di questa isobara, consideriamo il parallelo, il meridiano ed il vento di gradiente $\bar{v}$. Inoltre, sia a l'angolo che $\bar{v}$ forma con il nord geografico orientato positivamente da sud a nord.

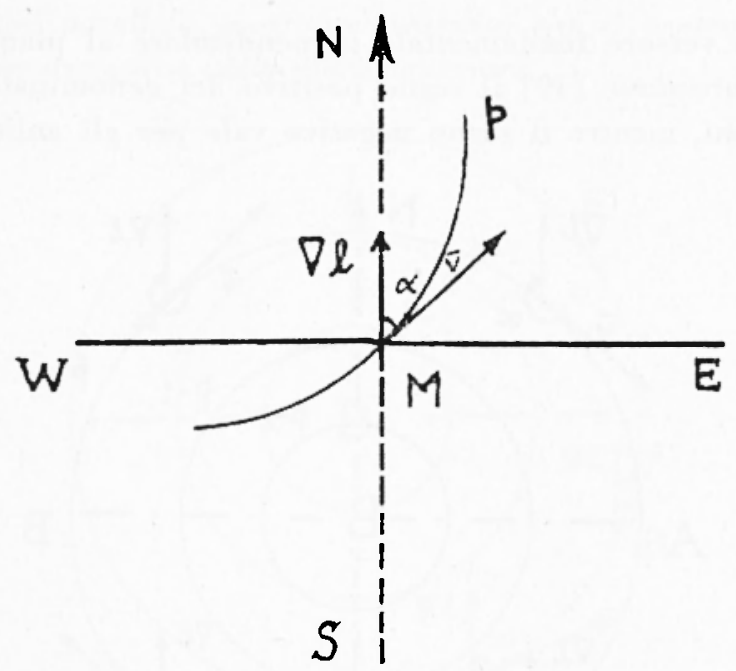

Fig. 1

11 vettore $\nabla l$ è disposto lungo il meridiano ed $\dot{e}$ diretto verso le alte latitudini. La grandezza del prodotto vettoriale $v \wedge \vee l$ è espressa da:

$$
v|\nabla l| \cdot \operatorname{sen} \alpha
$$

dove $|\nabla l|$ i la grandezza del vettore $-l$. Essa è data da $d l / d n$, dove dn è l'elemento di meridiano. Ora, siccome l'arco di meridiano $d n \dot{e}$ espresso da $r d \varphi$, dove $r$ è il raggio della terra e $d \varphi$ è la variazione di latitudine corrispondente a $d n$, si ba:

$$
|\vee l|=\frac{d l}{a_{n}}=\frac{l}{r} \frac{d l}{d n}
$$

e, poiché $l=2 w$ sen $q$, risulta anche $d l / d q=2 w \cos \varphi$. Quindi :

$$
|\nabla \iota|=\frac{2 w \cos \varphi}{r}
$$


Pertanto, per quest'ultima relazione e per la definizione di prodotto vettoriale, si ottiene:

$$
\frac{\bar{v} \wedge \nabla l}{l+2 \frac{v}{R}}=\frac{\operatorname{sen} \alpha}{r} \cdot \frac{2 \omega v \cos \varphi}{l+2 \frac{v}{R}} \bar{k}=\frac{\operatorname{sen} \alpha}{r} \frac{\omega v \operatorname{ccs} \varphi}{\omega \operatorname{sen} \varphi+\frac{v}{R}} \bar{k}
$$

dove $\bar{k}$ è il versore fondamentale perpendicolare al piano di $\bar{v}$ e di $\checkmark l$. Nell'espressione [19] il segno positivo del denominatore vale per le depressioni, mentre il segno negativo vale per gli anticicloni.

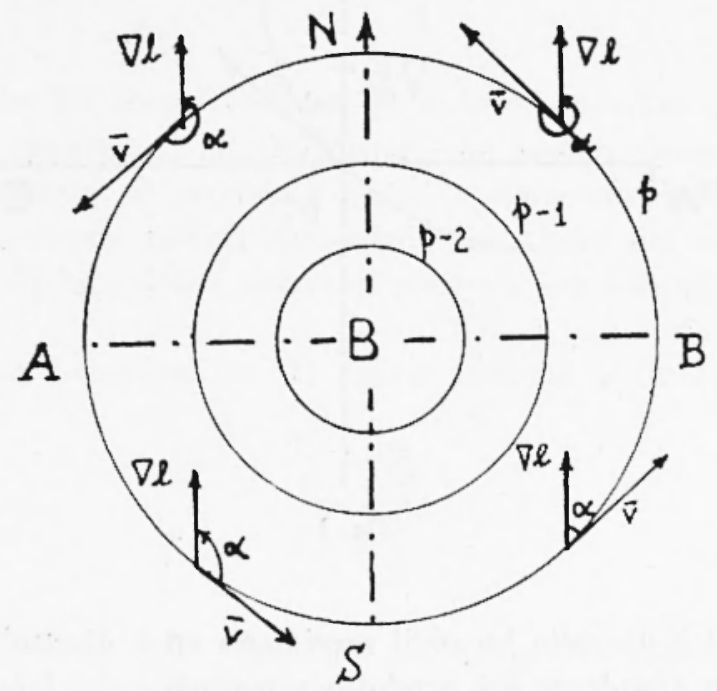

Fig. 2

Per avere un'idea del modo con cui la latitudine influisce sul segno del rotore del vento di gradiente, consideriamo un campo particolare della pressione costituito da una depressione ad isobare circolari e concentriclee, per cui $R$ è costante (fig. 2 ).

Per i punti situati sull'isobara $p$, sul ramo $A S B$ della metà inferiore di questa depressione, cioè sul ramo d'isolsara situato a sud del parallelo geografico passante per il centro depressionario, come si vede dalla figura, sen $a$ è sempre positivo. Il secondo membro della [19], dunque, è positivo in questo settore, essendo positive tutte le altre grandezze che vi compaiono. Conseguentemente, il rotore dovito all'effetto di latitudine è positivo, cioè ciclonico. Al contrario, nella metà superiore della depressione il rotore è negativo, ciò̀ anticiclo- 
nico. Nel caso di un anticiclone, tenuto conto del fatto che $w \operatorname{sen} \varphi-v / R$ è sempre positivo in un anticiclone, la distribuzione del rotore ̀̀ inversa.

Pertanto, possiamo concludere:

L'effetto di latitudine dà luogo ad un rotore positivo, ciò̀ a moto rotazionale ciclonico, nella metà inferiore di una depressione circolare, a sud del parallelo geografico passante per il centro, e a moto rotazionale anticiclonico nella metà superiore.

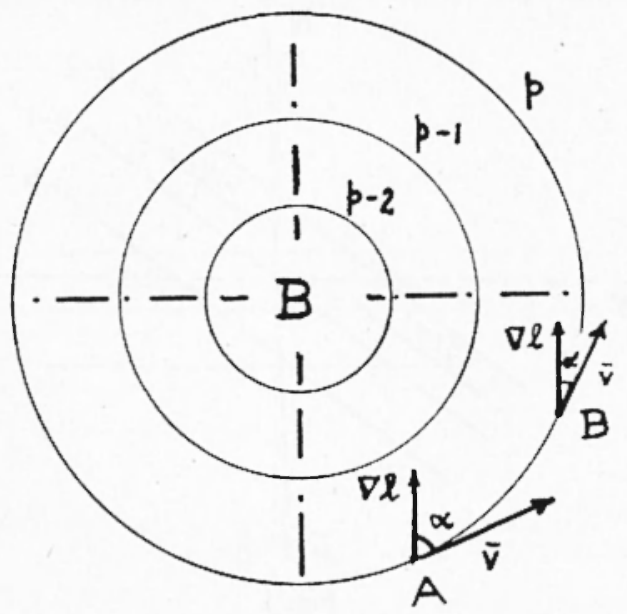

Fig. 3

Questa distribuzione del rotore per effetto della latitudine può griustificare presumililmente la formazione di circolazioni cicloniche secondarie a sud delle depressioni principali.

Per vedere ora come varia la grandezza del rotore quando varia la latitudine, consideriamo due casi particolarmente semplici.

(1) Supponiamo, in primo luogo, che il campo barico sia costituito, come prima, da isobare circolari e concentriche (fig. 3). Consideriamo i due veltori vento (di gradiente) nei punti $A$ e $B$ di $p$, appartenenti entrambi al quarto quadrante, per i quali sen $u>0$ e rot $v>0$. Osserviamo subito che sen $\alpha$ i decrescente dal punto $A$ al punto $B$.

Si sa, inoltre, che, nel caso di una depressione, il vento di gradiente è espresso da

$$
2 \text { a) } v \operatorname{sen} \text { iे }+\frac{v^{-}}{R}=\frac{G}{g}
$$


Il termine $G / \varrho$ può considerarsi costante, quando si suppone $B$ non troppo lontano da $A$. Ora, poiché sen $\varphi$ cresce da $A$ a $B, v$ deve diminuire. Quindi, anche in questo caso $v$ varia in modo inverso a $\varphi$. D'altra parte, facendo crescere $\varphi$ si vede subito che la [19] è decrescente. Conseguentemente: per moti rotazionali ciclonici che si svolgono da sud a nord, nella regione del piano a sud del parallelo passante per il centro, il rotore è decrescente. Per gli anticicloni vale la distribuzione contrariu.

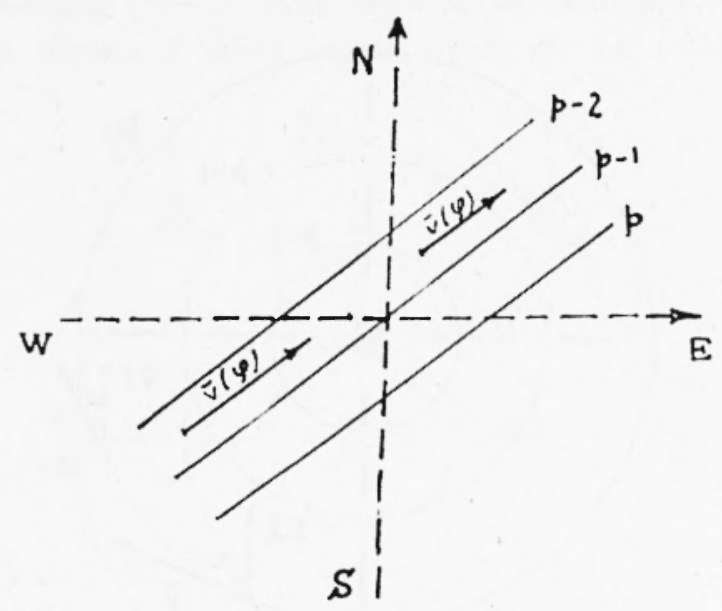

Fig. 4

b) Supponiamo, ora, che il campo barico sia costituito da isobare rettilinee $(1 / R=0)$, parallele ed equidistanti $(\bar{G}$ costante $)$ ed egualmente inclinate rispetto ai meridiani ( $\operatorname{sen} \alpha=$ costante) (fig. 4). In tal caso, la [19] diventa:

$$
\frac{\bar{v} \wedge \nabla l}{l \_2 \frac{v}{R}}=\frac{\operatorname{sen} \alpha}{r} \cdot v \cdot \operatorname{ctg} \varphi \bar{k}
$$

Il vento relativo a questa configurazione barica è il vento geostrofico, per cui vale la seguente relazione:

$$
v=\frac{G}{2 \omega \varrho \operatorname{sen} \omega}
$$

dove $G$ è la grandezza del gradiente barico. Ora, poiché $G$ è costante lungo l'isobara $p$, supponendo che nel tratto in esame la densità $Q$ non 
vari, varierà solo la latitudine $\varphi$. Per conseguenza, la grandezza $v$ della velocità varia in modo inverso a $\varphi$, cioè, $v$ decresce, quando aumenta $\varphi$. D'altra parte, nella $[20]$ anche cotg $\varphi$ decresce, quando $\varphi$ aumenta. Quindi nell'ipotesi sen $a<0$, possiamo concludere, come prima: per molti rettilinei che si svolgono da latitudini inferiori a latitudini superiori il rotore è ciclonico ma decrescente. Per moti atmosferici rettilinei che si svolgono da latitudini superiori a latitudini inferiori, il rotore $\dot{e}$ anticiclonico ma decrescente (").

Un caso particolare d'importanza notevole è la distribuzione del rotore in seno alle correnti occidentali rettilinee delle medie latitu-

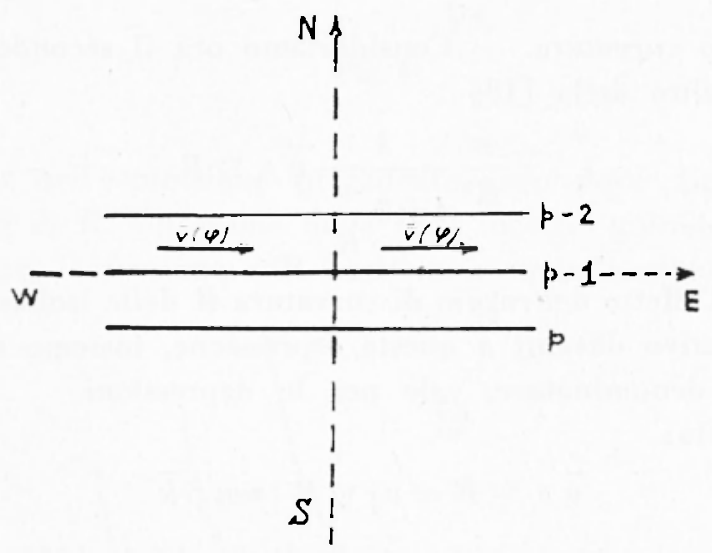

Fig. 5

dini dell'emisfero nord. Questo caso corrisponde bene alla cosiddetta " corrente a getto " (fig. 5 ).

In tal caso, osserviamo che $\bar{v}$ è perpendicolare a $(\bar{V} l)$ per cui la $[20]$ diventa:

$$
\frac{\bar{v} \wedge \backsim l}{l \pm 2 \frac{v}{R}}=\frac{v}{r} \operatorname{ctg} \varphi \bar{h}
$$

É noto che la corrente a getto subisce spostamenti di latitudine, abbassandosi verso latitudini più basse nei mesi invernali e portandosi a latitudini più elevate nei mesi estivi. Il rotore di tale corrente, come risulta dalla [21], è ciclonico e aumenta con il diminuire della

(*) Questo risultato trova immediata applicazione per le saccature costituite da isobare rettilinee e per $\mathbf{i}$ settori caldi delle depressioni in formazione, anch'essi costituiti da isobare rettilinee e parallele. 
latitudine $\varphi$ della corrente stessa. Si ha, cosi, una giustificazione indiretta dell'aumento dell'attività ciclonica nei mesi invernali, al di sotto di tale corrente.

Un altro caso particolare ma d'importanza più limitata è quello delle correnti che si svolgono lungo i meridiani geografici. In tal caso, poiché $\bar{v} \grave{e}$ parallelo a $\nabla l$, il rotore è nullo. Anche questo risultato giustifica l'osservazione che vari autori hanno rivelato in pratica e, cioè, che correnti di tale genere tendono a diventare curvilinee piuttosto che a sviluppare un movimento rotazionale in seno ad esse; precisamente, si sviluppa una curvatura ciclonica per moti rettilinei che si svolgono da nord a sud e curvatura anticiclonica nel caso opposto.

5. Effetto curvatura. - Consideriamo ora il secondo termine al secondo membro della [18]

$$
\overline{R^{2}} \frac{v}{l \pm 2 \frac{v}{R}} \bar{v} \wedge \nabla R
$$

che esprime l'effetto del raggio di curvatura $R$ delle isobare sul rotore. Il segno negativo davanti a questa espressione, insieme con il segno positivo del denominatore, vale per le depressioni.

Ora risulta:

$$
\bar{v} \wedge \nabla R=v|\nabla R| \operatorname{sen} \beta \bar{k}
$$

dove $\beta$ è l'angolo compreso fra $\bar{v}$ e $\nabla R$. Sicché la [22] diventa:

$$
\mp\left(\frac{v}{R}\right)^{2} \frac{1}{l \pm 2 \frac{v}{R}}|\nabla R| \operatorname{sen} \beta \bar{k}
$$

Come è ovvio, risulta sen $\beta>0$, quando $0<\underline{\beta}<180$. Questa condizione è valida sia che $R$ cresca nella direzione di $\bar{v}\left(90^{\circ}<\beta<180^{\circ}\right)$, sia che $R$ decresca in questa direzione $(90<\beta<180)$.

L'espressione [23] mostra, pertanto:

Nelle depressioni, il rotore dovuto alla curvatura delle isobare, indipendentemente dall'intensità di questo, è sempre anticiclonico. Negli anticicloni il rotore $\dot{e}$ sempre ciclonico.

Per quanto riguarda la variazione della grandezza del rotore al variare di $R$, riferiamoci al caso per il quale l'isobara in esame appartenga ad una depressione e facciamo astrazione dall'effetto di latitudine ( $p=$ costante). In queste condizioni, il termine

$$
2 \omega v \operatorname{sen} \varphi+\frac{v^{2}}{R}
$$


¿̀ costante, perché, come prima, è costante $G / \varrho$. Si vede, dunque, che, quando $R$ diminuisce, deve diminuire anche $v$; ma, poiché $v$ compare
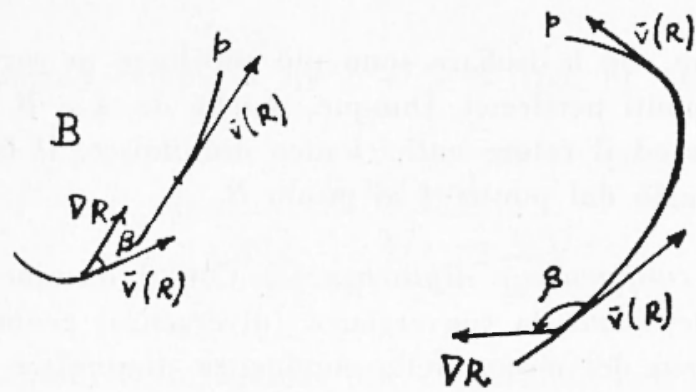

Fig. 6

al quadrato nell'espressione precedente, esso deve diminuire meno rapidamente di $R$. Un esame della [23] mostra, quindi, che, quando $R$ diminuisce, la frazione $v / R$ aumenta e, quindi, aumenta il rotore

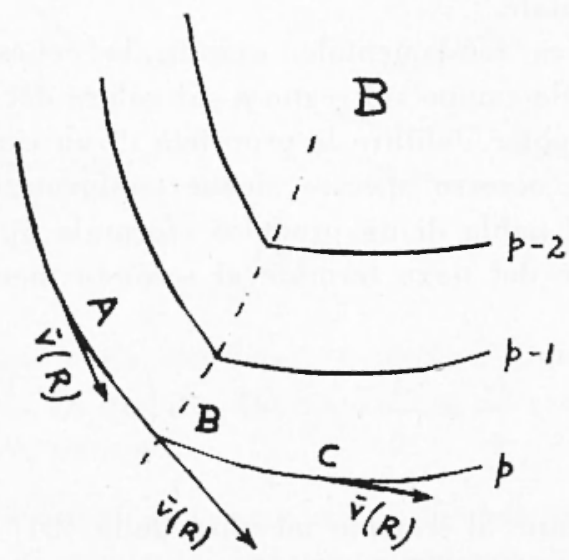

Fig. ?

dovuto al raggio di curvatura. Questo risultato trova un'applicazione immediata per le saccature e per i promontori.

Per quanto riguarda le saccature, consideriamo i tre punti $A, B$ e $C$ situati a sinistra, sull'asse e a destra della saccatura rispettivamente (fig. 7). Molto spesso accade che, quando ci si sposta nello stesso verso del vento, il raggio di curvatura $R$ cresce dal punto $A$ al punto $B$ e decresce da $B$ a $C$. 
Si ha, in altre parole $\left({ }^{1}\right)$ :

$$
R_{\mathrm{A}}<R_{\mathrm{B}}<R_{\mathrm{C}}
$$

il che vuol dire che le isobare sono più rettilinee in corrispondenza di $B$ che nei punti periferici. Dunque, poiché da $A$ a $B$ il ragroio $R$ cresce, cresce $v$ ed il rotore anticiclonico diminuisce. Il contrario accade nel passaggio dal punto $A$ al punto $B$.

6. Effetto confluenza e diffluenza. - Con il termine confluenza (diffuenza) intendiamo la converwenza (divergenza) geometrica delle isobare nel verso del moto. Nella confluenza diminuisce la distanza lungo la normale alle isobare e, quindi, aumenta il gradiente barico.

È nota limportanza che ha assunto recentemente la cosidetta teoria della divergenza di Scherhag. Egli, riferendosi ad una forma confluente e diffluente del campo barico in quota, ha messo in evidenza notevoli proprietà di questo campo agli effetti della formazione di cicloni ed anticicloni doruti allo stabilirsi di campi di convergenza e di divergenza orizzontale.

E d'importanza fondamentale, quindi, la conoscenza dell'eventuale influsso di tale campo sul segno e sul valore del rotore del vento di gradiente. Per poter stabilire le proprietà di un campo di confluenza e di diffluenza, occorre operare alcune trasformazioni.

Ricordando il nabla di un prodotto (formula 5), trasformiamo il prodotto vettoriale del terzo termine al secondo membro della [18]. Si ha:

$$
\bar{v} \wedge \nabla \frac{G}{\varrho}=\frac{1}{\varrho} \bar{v} \wedge \nabla G+G\left(\bar{v} \wedge \nabla \frac{1}{\varrho}\right)
$$

Il primo termine al secondo membro della [24] fornisce l'effetto della confluenza e della diffluenza sul rotore. Questo effetto, pertanto, è espresso da:

$$
\pm \frac{\bar{v} \wedge \nabla G}{\varrho R\left(l \pm \frac{v}{R}\right)\left(l \pm 2 \frac{v}{R}\right)}
$$

(1) È implicita l'ipotesi che la curvatura delle isobare sia sempre ciclonica. Un'estensione dei risultati qui esposti si può ottenere da un esame dettagliato delle varie curvature che si presentano in pratica, per le saccature e per $i$ promontori. 
Il prodotto vettoriale che compare al numeratore ha per valore

$$
v|\nabla G| \operatorname{sen} \gamma
$$

dove $\gamma$ è l'angolo compreso fra $\bar{v}$ e $\nabla G$ (fig. 8). Quindi, la [25] diventa:

$$
\pm \frac{v|\nabla G| \operatorname{sen} \gamma}{\varrho R\left(l \pm \frac{v}{R}\right)\left(l \pm 2 \frac{v}{R}\right)}
$$

Il segno positivo corrisponde alle depressioni, mentre il segno negativo corrisponde agli anticicloni. Questo segno, come si vede dalla
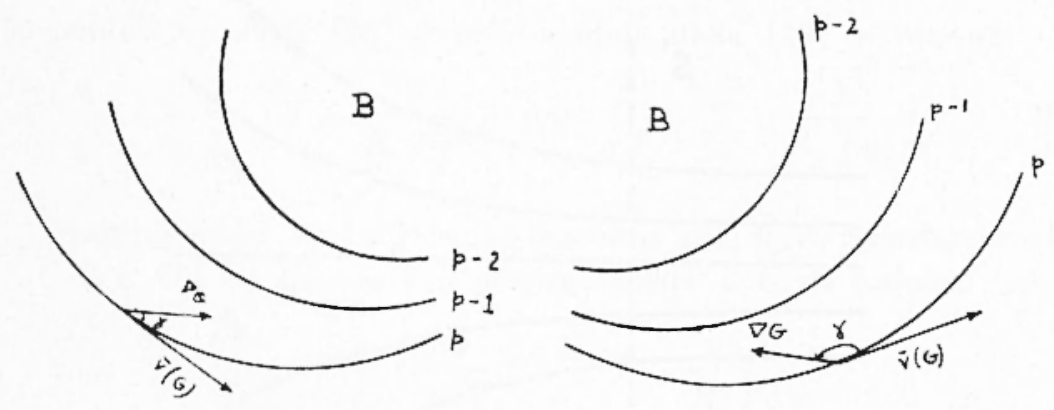

Fig. 8

figr. 8 , non dipende dalla confluenza o dalla diffluenza delle isobare di una depressione, in quanto, nei due casi, risulta sempre sen $\gamma>0$. $\mathrm{Si}$ può concludere, dunque:

nelle depressioni con zone di confluenza o di diffluenza, il rotore del vento di gradiente, indipendentemente dalla entitiz di questo, è sempre ciclonico. Negli anticicloni accude il contrario.

Prendiamo in considerazione ora un campo di diffluenza costituito da isolsare rettilinee che si aprono a ventaglio (fig. 9). Per il tratto rettilineo di queste correnti, il rotore è dato, come è noto, da:

$$
\frac{v}{r} \operatorname{ctg} q k
$$

Non appena si entra nel “ delta ", in corrispondenza della sezione $S S^{\prime}$, 
per effetto della diffluenza, questo valore muta in

$$
\frac{v|\nabla G| \operatorname{sen} \gamma}{\varrho R\left(l+\frac{v}{R}\right)\left(l+2 \frac{v}{R}\right)} \bar{k}
$$

per la zona $C$ a nord dell'isobara rettilinea $p$ e nel valore

$$
-\frac{v|-G| \operatorname{sen} \gamma}{\varrho R\left(l-\frac{v}{R}\right)\left(l-2 \frac{v}{R}\right)} \bar{k}
$$

per la zona $A$.

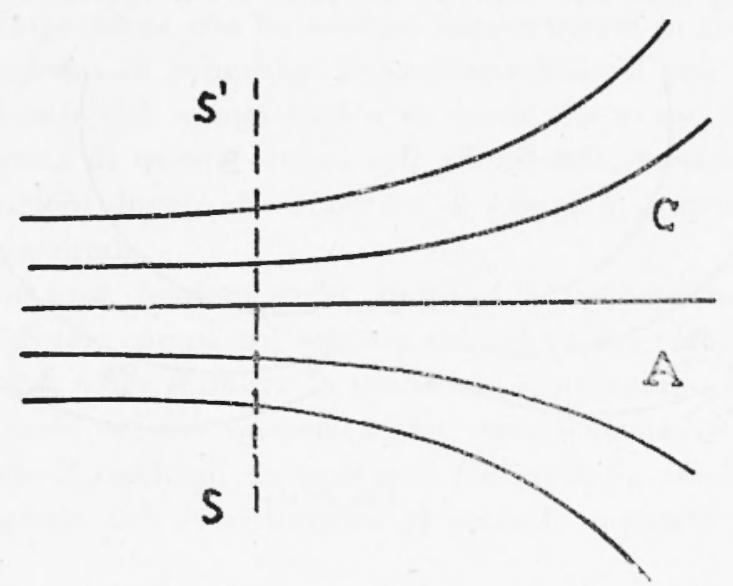

Fig. 9

7. Effetto avvezione della temperatura. - 11 secondo termine al secondo membro della [24] fornisce questo effelto. Dalla formula [18] risulta che questo effetto è dato da:

$$
\pm \frac{G\left(\bar{v} \wedge \nabla \frac{1}{n}\right)}{R\left(l+\frac{v}{R}\right)\left(l \pm 2 \frac{v}{R}\right)}
$$

Quest'espressione contiene $\nabla 1 / \varrho$ che non è possibile valutare direttamente in pratica, in quanto non vengono tracciate le carte isosteriche $(\varrho=$ costante). Pertanto, è necessario sostituire questo nabla con 
quello della temperatura, permettendoci di utilizzare le carte isotermiche.

Per ragrgiungere questo scopo, utilizziamo la nota relazione

$$
p=R_{\mathrm{\rho}} T
$$

dove $P$ è la pressione, $R_{\text {i }}$ la costante caratteristica dei gas e $T$ la lemperatura assoluta. Da questa equazione risulta:

$$
\neg p=R_{\text {แ }} \nabla T+R_{\mathrm{a}} T \nabla \varrho
$$

Ora

$$
\frac{1}{\varrho}=-\frac{70}{\varrho^{2}}
$$

Ricavando $\nabla$ e dalla [28] e sostituendola nella [29] si olliene:

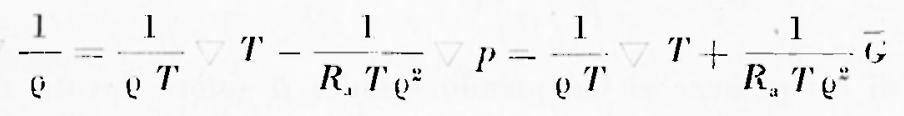

Moltiplicando la $[30]$ veltorialmente per $\bar{v}$ e ricordando che $v \wedge \bar{G}=v \cdot G \bar{l}$ in quanto $\bar{v} \dot{e}$ perpendicolare a $\bar{G}$, si ottiene:

$$
\bar{v} \wedge / \frac{1}{\varrho}-\frac{1}{\varrho T} \bar{v} \wedge \nabla T+\frac{v \cdot G}{\bar{n}_{1} \overline{\mathrm{T} Q^{2}}} \bar{k}
$$

Sostituendo nellat [2i] si ha:

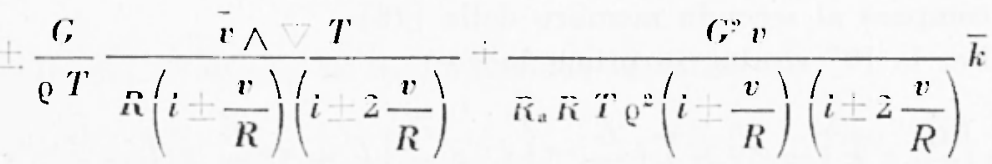

Questa i l'espressione del rotore dovuto all'eftetto arvetlivo delle isolerme. Il segno posilivo corrisponde alle depressioni ed il segno negrativo ayli anticicloni.

Consideriamo il caso delle depressioni. Il secondo termine che compare nella [31] è sempre positivo, in quanto il coefficiente del versore $\overline{i_{\text {, }}}$, essendo costituito da grandezze tutte positive, è positivo. Non altrettanto accarle per il primo termine, potendo il prodotto vetioriale assumere valore positivo o negativo a secondo che l'angolo $\delta$ compreso fra $\bar{v}$ e $\nabla T$ si mantiene inferiore o superiore a $180^{\circ}$. Questo termine, poi, si annulla quando $\bar{v}$ è parallelo a $\nabla T$. In quest'ultimo caso, che 
corrisponde all'avvezione massima, la famiglia di isoterme è perpendicolare alle isobare e compare solo l'effetto de secondo termine, vale a dire: nelle depressioni, l'avvezione massima di aria calda o fredda dà luogo sempre ad un rotore ciclonico. Il contrario accade per gli anticicloni. Quando l'avvezione non è massima ma $0<\delta<180^{\circ}$, si può concludere: nelle depressioni, l'avvezione di aria calda dà luogo a moto rotazionale ciclonico.

Nel caso in cui $\bar{v}$ e $\nabla T$ formano un angolo superiore a $180^{\prime \prime}$, non si può trarre alcuna conclusione, in quanto, in tal caso, il primo termine della [31] ’̀ negativo, mentre il secondo termine è positivo. Tuttavia, possiamo dire che il rotore che ne risulta è ciclonico, quando il secondo termine predomina sul primo. In tal caso, risulta:

$$
\frac{G}{p}>\frac{\nabla T}{T} \operatorname{sen} \hat{0}
$$

Il segno di eguaglianza si ha quando è nullo il rotore dovuto all'effetto termico avvettivo e viceversa. Ha, quindi, notevole importanza la rappresentazione del campo delle isoterme sovrapposto a quello del vento.

Un caso limite interessante si presenta quando le isoterme sono parallele a $\bar{v}$, nel qual caso si può osservare che, anche in assenza di solenoidi isobarico-isotermici (condizione di barotropia) il rotore esiste.

8. Effetto divergenza, ecc. - Occupiamoci ora dell'ultimo termine che compare al secondo membro della [18].

Per la [9] risulta, in primo luogo:

$$
\nabla \wedge\left(\frac{\bar{G}}{\varrho} \wedge \bar{k}\right)=(\nabla \times \bar{k}) \frac{\bar{G}}{\varrho}-\left(\nabla \times \frac{\overline{c_{i}}}{\varrho}\right) \bar{k}+\nabla \frac{\bar{G}}{\varrho} \times \bar{k}-\frac{\bar{G}}{\varrho} \times \nabla \bar{k}
$$

Osserviamo subito che il termine $\bar{V} \times \bar{h} \grave{e}$ la divergenza del veltore unitario $\bar{k}$. Ora, poiché $\bar{k}$ è un vettore costante fintensità uno, direzione e verso costantel, la sua divergenza $\dot{e}$ nulla, ciò̀, $\nabla \times \bar{k}=0$. E facile accertarsi, inoltre, che l'espressione precedente si riduce a $\left({ }^{1}\right)$ :

$$
\nabla \wedge\left(\frac{\bar{G}}{\varrho} \wedge \bar{k}\right)=-\left(\nabla \times \frac{\bar{G}}{\varrho}\right) \bar{k}
$$

(1) Questa relazione è immediata, quando si sviluppano i determinanti simbolici che rappresentano il doppio prodotto vettoriale che compongono questo termine. 
Ora, per la $[10]$, dove $f=1 / 0$ e $v=\bar{G}$, si ha:

$$
\nabla \times \frac{\bar{G}}{\varrho}=\frac{1}{\varrho}(\nabla \times \bar{G})+\nabla \frac{1}{\varrho} \times \bar{G}
$$

Analogamente a quanto si é fatto per l'effetto avveltivo termico, i può far comparire al secondo membro di quest'espressione il vettore gradiente della temperatura, utilizzando la relazione:

$$
\nabla \frac{1}{\varrho}=\frac{\nabla T}{\varrho T}: \frac{\bar{G}}{R_{\mathrm{a}} T \varrho^{2}}
$$

Quindi, ricordando che

$$
\bar{G} \times \bar{G}=G^{2}
$$

si ottiene:

$$
\nabla \frac{1}{\varrho} \times \bar{G}=\frac{1}{\varrho \bar{i}}(\nabla T \times \bar{G})+\frac{G^{2}}{R_{\mathrm{u}} \bar{T} p^{2}}
$$

e la $[32]$ diventa:

$$
\nabla \times \frac{1}{\varrho} \overline{\bar{G}}=\frac{\nabla \times \bar{G}}{\varrho}+\frac{\nabla T \times \bar{G}}{\varrho T}+\frac{G^{2}}{R_{\mathrm{a}} T Q^{2}}
$$

Infine, si oltiene la trasformazione cercata:

$$
\nabla \wedge\left(\frac{\bar{G}}{\varrho} \wedge \bar{k}\right)=-\frac{1}{\varrho}\left[\nabla \times \bar{G}+\frac{\nabla T \times \bar{G}}{T}+\frac{G^{2}}{R_{\mathrm{a}} T \varrho}\right] \bar{k}
$$

Ouindi :

$$
\frac{\nabla \wedge\left(\frac{G}{\varrho} \wedge \bar{k}\right)}{l+\frac{v}{R}}=-\left[\frac{\nabla \times \bar{G}+\frac{1}{T} \nabla T \times \bar{G}+\frac{G^{2}}{R_{\mathrm{a}} T_{Q}}}{\varrho\left(l \pm \frac{v}{R}\right)}\right] \bar{k}
$$

I risultati che sequono valgono sia per $\mathrm{i}$ cicloni che per gli anticicloni. 
L'effetto della divergenza del vettore gradiente è espresso dal primo termine a secondo membro della $[33]$ :

$$
-\frac{\nabla \times \bar{G}}{\varrho\left(l \pm \frac{v}{R}\right)} \bar{k}
$$

Se questo vettore diverge $(\nabla \times \bar{G}>0)$ il rotore $\dot{e}$ anticiclonico, perché vale il segno negativo davanti al termine suddelto; se questo termine converge $(\nabla \times \bar{G}<0)$ il rotore è ciclonico.

Il secondo termine

$$
-\frac{\Gamma T \times \bar{G}}{\varrho T\left(l \pm \frac{v}{R}\right)} \bar{k}=-\frac{G}{\varrho T\left(l \pm \frac{v}{R}\right)} \cdot \frac{\partial T}{\partial n} \bar{k}
$$

esprime l'effetto isoharico-isotermico, dove $\partial T / \partial n$ i la variazione della temperatura $T$ lungo la normale alle isohare.

Se il campo delle isoterme è orientato rispetto al gradiente barico in modo che il gradiente termico formi un angolo inferiore a $90^{\circ} \mathrm{o} \mathrm{su-}$ periore a $270^{\circ}$ con $\bar{G}$, allora il rotore è ciclonico.

Ritornando al caso in cui le isoterme di una depressione siano parallele alle isoluare (condizione di harotropia) e decrescenti verso il centro depressionario (vortice freddo), risulta $\nabla T$ opposto a $\bar{G}$ ed il prodotto scalare $\nabla T \times \bar{G}=-|\nabla T| G$. In tal caso, si è già visto che il rotore è ciclonico. Anche il termine in esame diventa positivo, per cui il rotore è ciclonico.

Per quanto riguarda l'ultimo termine costante, si può osservare clie esso è sempre negativo e potrebbe, in un certo senso, compensare l'effetto degli altri due. La determinazione in pratica dell'effetto dei singoli termini può essere agevolata dall'uso di abaci di immediato impiego. Da questi abaci, già determinati da Vialar ( ${ }^{1}$, si possono calcolare i coefficienti dei prodotti vettoriali dei singoli termini.

Questi risultati confermano, in pratica, l'osservazione che non i possibile dare "a priori " una stima del valore del rotore, poiché tutti i fattori precedentemente considerati sono suscettibili di agire contemporaneamente. Tuttavia, possiamo dare un'idea dell'ordine di grandezza massima del rotore risultante da questa combinazione, alle nostre latitudini e nei hassi strati dell'atmosfera.

(1) Vialar J., La Convergence et Divergence, Con/luence et dillluence. Journ. Scient. Météor., n. 3, 1951. 
Si ottiene la seguente tabella:

\begin{tabular}{|c|c|c|c|c|c|c|c|}
\hline $\begin{array}{c}\text { Effetto } \\
\text { massimo }\end{array}$ & if & $\mathrm{R}$ & & 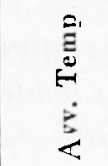 & Div. & 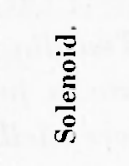 & 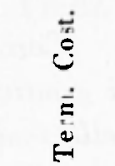 \\
\hline B. & $0,2.10^{5}$ & טי & $0,3.10=$ & $0,1.10$, & י & ני & $0,21 \cdot 10^{-4}$ \\
\hline A. & $0,4.10 \div$ & בَ-10,2.10 & $2,5.10 \cdot$ & "0,3.10 & נ, נ, & o,45.10 & $0,61.10^{-4}$ \\
\hline
\end{tabular}

I valori compresi in questa tabella sono stati ricavati dai seguenti dati:

1) Alla distanza di circa $600 \mathrm{~km}$ dal centro depressionario si è assunto come intensità del vettore gradiente barico il valore $3,5 \mathrm{mh}$ per $100 \mathrm{~km}$. Per gli anticicloni, invece, si è assunto un valore di $0,5 \mathrm{mb}$.

2) Raggio di curvatura eguale a $300 \mathrm{~km}$; variazione di raggio di curvatura di $10 \mathrm{~km}$ per $100 \mathrm{~km}(\partial R / \partial n=0,1)$; densità $\varrho=1,29310^{3}$ (C.G.S.), per la latitudine di 45".

3) Variazione del gradiente barico $(\partial G / \partial n)$ eguale a $310^{-12}$ harie. cm-cm. Valore di $G$ eguale a $510^{\circ}$ (C.G.S.); $T=285^{\circ}$. Valore di $2 T / 2 n$ equale a $210^{-7}$ gradi $\mathrm{cm}^{-1}$.

Da questa tabella risultano le seguenti caralteristiche:

1) Nelle depressioni i vari effetti si equivalgono come ordine di grandezza fatla eccezione del termine noto.

2) Negli anticicloni l'effetıo della confluenza e della diffluenza è neltamente predominante sugli altri ed assume importanza la divergenza del gradiente barico orizzontale.

L'esposizione del presente lavoro ed i relativi calcoli seguono la traccia di uno studio analogo sulla convergenza e la divergenza dell'atmosfera di Vialar, J. (vedi nota bibliografica a pag. 20), allo scopo di rendere immediato il confronto dei risultati delle analisi delle due fra le più importanti grandezze in meteorologia.

Roma - Ispettorato Telecomunicazioni Assistenza al VoloMinistero della Difesa - Aeronatica - Giugno 1951. 


\section{RIASSSUNTO}

Con l'ausilio dell'analisi vettoriale si esprime il rotore del vento di gradiente in funzione del " nabla" della latitudine, della curvatura delle isobare, della temperatura e del gradiente barico orizzontale. Si determina, per ciascun fattore, la variazione in grandezza e segno del rotore del vento di gradiente e si determina il rotore in alcuni casi particolari, fra $i$ quali la cosiddetta corrente a getto.

I risultati di questa analisi mostrano alcune proprietà notevoli del rotore del vento di gradiente, non evidenti "a priori", che rendono più chiaro questo importante ramo della cinematica dell'atmosfera.

\section{$S U M M A R Y$}

With the help of vector analysis, the curl of the gradient wind is expressed as a function of four variables: the "nabla" of latitude, curvature of isobars, temperature and horizontal pressure gradient. For each of these variables the variation of intensity and sign of the curl of the gradient wind is discussed. The curl is then analysed and determined for special cases and for the jet stream.

The results of this analysis show some relevant proprieties of the curl of the gradient uind, which lead to a better understanding of this important branch of kinematics of the atmosphere. 